Procedimiento de optimización no lineal para la cuantificación del aporte de la energía eléctrica en el crecimiento económico colombiano, 1925-1997

Oscar Gonzalo Manrique-Díaz y Diego Fernando Lemus-Polanía 
Procedimiento de optimización no lineal para la cuantificación del aporte de la energía eléctrica en el crecimiento económico colombiano, 1925-1997

Resumen: En este trabajo se incluye la energía eléctrica (E) como una tercera variable en la función de producción estándar Cobb-Douglas para determinar su participación en el PIB de Colombia y calcular su elasticidad asociada entre 1925 y 1997. Para tal efecto, se emplean el modelo LINEX y el algoritmo de optimización no lineal de Levenberg-Marquardt, bajo el supuesto de elasticidades de producción no negativas para ajustar la función de producción, corregida y basada en dicho modelo. El análisis se alimenta de las series de PIB, capital (K) y trabajo (L) que elaboró el GRECO para el Banco de la República, y también de una serie temporal de energía eléctrica (E) producida con el patrocinio de Colciencias. Los resultados calculados para las elasticidades asociadas permiten verificar que, en concordancia con los casos de estudio conocidos, la energía eléctrica ba tenido mayor peso en la estructura del PIB que los factores tradicionales. Se concluye que la condición de rendimientos constantes de la función de producción estándar no se aplica a ninguna economía conocida, sea esta de un país desarrollado o no.

Palabras clave: crecimiento económico; elasticidad de un factor; función de producción LINEX; procedimiento de ajuste no lineal.

Clasificación JEL: O47, A10, C33, C61.

Nonlinear optimization method for quantifying the contribution of electricity in the Colombian economic growth, 1925-1997

Abstract: In this work, electrical energy (E) is included as a third variable in the Cobb-Douglas standard production function, to determine its participation in Colombia's GDP and calculate its associated elasticity between 1925 and 1997. For this purpose, the LINEX model and the LevenbergMarquardt nonlinear optimization algorithm was used, under the assumption of non-negative production elasticities, to adjust the production function, corrected and based on said model. The analysis is fed by the GDP, capital (K) and labor (L) series prepared by GRECO for Banco de la Repuiblica, and also by a electrical energy (E) time series produced under the sponsorship of Colciencias. The results calculated for the associated elasticities allow to verify that, in accordance with the known case studies, electrical energy has had a greater weight in the structure of GDP than traditional factors. It is concluded that the constant returns condition of the standard production function does not apply to any known economy, whether it is from a developed country or not.

Keywords: economic growth; elasticity of a factor; LINEX production function; nonlinear fitting procedure.

Este artículo y sus anexos se distribuyen por la revista Lecturas de Economía bajo los términos de la Licencia Creative Commons Atribución-NoComercial-CompartirIgual 4.0. https://creativecommons.org/licenses/by-nc-sa/4.0/ 
Optimisation non linéaire pour la quantification de la contribution de l'énergie électrique á la croissance économique colombienne, 1925-1997

Résumé: Dans ce travail, l'énergie électrique (E) est incluse comme troisiéme variable dans la fonction de production standard du type Cobb-Douglas, afin de déterminer sa participation au PIB de Colombie et pouvoir ainsi calculer son élasticité associée entre 1925 et 1997. Pour ce faire, nous utilisons le modéle LINEX et l'algorithme d'optimisation non linéaire de Levenberg-Marquardt, sous l'hypothése d'élasticités de production non négatives. Cette démarche permet d'ajuster et de corrigées la fonction de production. L'analyse est basée sur les séries du PIB, capital (K) et travail (L) que les chercheurs du GRECO ont préparé pour le Banco de la República, ainsi que sur une série cbronologique d'énergie électrique (E), laquelle est obtenue grâce au parrainage de Colciencias. Les résultats calculés pour les élasticités associées permettent de vérifier que, conformément aux études déjà connues, l'énergie électrique a eu un poids plus important dans la structure du PIB par rapport aux facteurs traditionnels. Nous concluons que la condition de rendement constant de la fonction de production standard ne s'applique á aucune économie connue, qu'elle soit ou non développée.

Mots clés: croissance économique; élasticité d'un facteur; fonction de production LINEX; procédure d'ajustement non linéaire.

Cómo citar / How to cite this item:

Manrique-Díaz, O. G. \& Lemus-Polanía, D. F. (2020). Procedimiento de optimización no lineal para la cuantificación del aporte de la energía eléctrica en el crecimiento económico colombiano, 1925-1997. Lecturas de Economía, 93, 65-100.

https://doi.org/10.17533/udea.le.n93a341714 


\title{
Procedimiento de optimización no lineal para la cuantificación del aporte de la energía eléctrica en el crecimiento económico colombiano, 1925-1997
}

\author{
Oscar Gonzalo Manrique-Díaz (iD ${ }^{\mathrm{a}}$ y Diego Fernando \\ Lemus-Polanía
}

-Introducción. -I. Los factores de producción y su agregación. -II. Funciones de producción consideradas. -III. Discusión de resultados. -Conclusiones. -Agradecimientos. -Referencias.

Primera versión recibida el 20 de diciembre de 2018; versión final aceptada el 13 de abril de 2020

\section{Introducción}

En los análisis del funcionamiento de las sociedades modernas, es de común aceptación que la energía es un factor determinante de todas las actividades económicas, productivas y sociales; de hecho, la energía puede ser considerada como el elemento clave en el proceso evolutivo y cultural del hombre (White, 1943). Después de siglos de uso de la fuerza del trabajo de los hombres y animales y del uso productivo de recursos como el agua, el viento y la madera, desde la primera fase de la Revolución Industrial se incrementó aceleradamente el uso de recursos como el carbón, el petróleo y el gas natural necesarios para crear y mover los nuevos esclavos mecánicos, construir modernas sociedades industrializadas y crear circuitos de comercio de alcance global. En dos siglos y medio, fue posible acelerar el proceso de creación de riqueza y lograr avances importantes en materia económica y social para la mayoría de las sociedades existentes.

a Oscar Gonzalo Manrique-Díaz: profesor asociado al Departamento de Economía, Facultad de Ciencias Humanas y Económicas, Universidad Nacional de Colombia - Sede Medellín, Colombia. Dirección postal: Universidad Nacional de Colombia - Sede Medellín, calle 59a \#63-20, oficina 403. Dirección electrónica: ogmanriq@unal.edu.co

https://orcid.org/0000-0001-8253-0539

b Diego Fernando Lemus-Polanía: investigador, Facultad en Estadística, Universidad Santo Tomás, Bogotá D.C., Colombia. Dirección postal: carrera 9 \#51-11, tercer piso, Facultad de Estadística. Dirección electrónica: dflemusp@gmail.com

https://orcid.org/0000-0002-6336-9636 
Manrique-Díaz y Lemus-Polanía: Procedimiento de optimización no lineal...

El intenso consumo de energía aceleró la creación de riqueza (simbolizada económicamente en el PIB), consolidó el sistema capitalista y elevó al crecimiento económico al status de uno de los principales campos de análisis teórico de la economía. Dicha aceleración fue estudiada y modelada por Solow $(1957)^{1}$ quien concluyó que la riqueza de los EE. UU. era explicada marginalmente por la combinación del capital (K) y el trabajo (L), y que la mayor parte de dicho crecimiento quedaría asociada al denominado residuo de Solow, el cual, para darle consistencia matemática al modelo fue explicado como un factor exógeno y tratado como una función del tiempo. Posteriormente dicho residuo se identificó con la productividad total de los factores (PTF), la tecnología en general (A), y más comúnmente con el progreso técnico. Con todo, y tal como este mismo autor reconocería después, se había formulado una teoría del crecimiento a la vez que se había dejado sin explicación el factor más importante (Solow, 1994, p. 48).

En este contexto, la teoría económica ortodoxa ha negado sistemáticamente que pueda existir una relación entre crecimiento económico y energía; en lo fundamental se argumenta que la energía es un bien intermedio que se produce y se consume en el proceso económico, cuyo peso en el producto final es marginal y es proporcional a su participación en la estructura de costos de producción. Sin embargo, desde los años setenta del siglo XX y como respuesta a la importancia que la energía lograba en la agenda de investigación económica como consecuencia de experiencia acumulada y posterior a las llamadas 'crisis' petroleras, el interés de los académicos e investigadores de diferentes disciplinas por establecer y formalizar dicha relación fue tomando forma y después de un largo proceso, en la literatura se reconocen dos grandes metodologías para aproximarse al análisis y las implicaciones de esta relación.

En primer lugar y desde una perspectiva ortodoxa, a partir del trabajo seminal de Kraft y Kraft (1978), se establece una relación estadística de causalidad entre el crecimiento del PIB y el consumo de energía. El camino abierto por estos autores ha sido seguido en numerosos trabajos que incluyen diversas variaciones analíticas y estadísticas, los cuales suelen ser realizados

1 Cabe recordar que este trabajo tenía como base el artículo seminal de Cobb y Douglas (1928), a partir del cual y en función de información estadística agregada y de alta calidad se sentarían las bases de la teoría estándar del crecimiento económico. 
con variaciones analíticas y estadísticas para diferentes países y regiones con resultados normalmente disímiles. Entre dichos trabajos se pueden reseñar los siguientes: Akarka y Long (1980), Yu y Choi (1985), Nachane et al. (1988), Hwang y Gum (1991), Ebohon (1996), Cheng (1997), Asafu-Adjaye (2000), Soytas y Sari (2003), Oh y Lee (2004), Shiu y Lam (2004), Altinay y Kagarol (2004), Yoo (2006), Lee (2006), Lee y Chang (2007), Squalli (2007), Chiou et al. (2008), Chontanawat et al. (2008), Yuan et al. (2008), Belloumi (2009), WoldeRufael (2009), Apergis y Payne (2010a, 2010b), Ozturk et al. (2010), Sharma (2010), Belke et al. (2011), Shuyun y Donghu (2011), Bildirici y Kayicky (2012), Gurgul y Lach (2012), Narayan y Popp (2012), Yalta y Cakar (2012), Yildirim y Aslan (2012), Zhang y Xu (2012), Shengfeng et al. (2012), Farhani y BenRejeb (2012). En el caso específico de Colombia la literatura existente registra los trabajos de Campo y Sarmiento (2011) y Barreto y Campo (2012).

La segunda metodología que se puede denominar heterodoxa, se deriva de los trabajos de autores ${ }^{2}$ que han buscado incorporar a la energía (E) como una variable adicional al capital $(\mathrm{K})$ y al trabajo $(\mathrm{L})$ en la función de producción estándar (Cobb-Douglas), desarrollando una metodología que sin alterar los fundamentos de la teoría neoclásica permitieran lograrlo con arreglo al mundo físico y sus leyes, de tal manera que también fuera posible calcular su aporte a la creación de riqueza, en función de la elasticidad asociada y poder medir en el tiempo su contribución al PIB. Entre los primeros intentos para lograr este propósito, cabe recordar a Berndt y Wood (1975), Allen (1979), Hannon y Joyce (1981) y Stern (1993, 2004). Con mejores resultados y buscando tender puentes para conciliar el mundo teórico de la economía con el mundo real de la física y por extensión de la biofísica, los trabajos de Kümmel (1980, 1982, 1989, 2011), Kümmel y Lindenberger (2014), Kümmel et al. (1985, 2000, 2002, 2010, 2015), Hall et al. (2001) y Lindenberger et al. (2017) han construido una metodología completa y efectiva que ha evolucionado para convertirse en una

2 Desde fines del siglo XIX diferentes autores han buscado métodos alternativos para explicar la creación de la riqueza económica y sus relaciones con la energía y sus leyes, apelando a diversas disciplinas y en función de diversos enfoques: Autores como Ostwald (1907), Soddy (1912), Lotka (1922), Tyron (1927), Henderson (1933), Cottrell (1955), hicieron importantes aportes históricos y conceptuales. Trabajos más recientes son los de Schurr y Netschert (1960), Jensen (1970), Georgescu-Roegen (1971, 1975), Kraft y Kraft (1978) y Jorgenson $(1978,1981,1984)$. 
Manrique-Díaz y Lemus-Polanía: Procedimiento de optimización no lineal...

alternativa analítica que permite analizar de manera alternativa el crecimiento económico, eliminar el residuo de Solow, hacer entendible el problema de la eficiencia tanto en la producción como en el consumo y abrir debates relativos a los impactos sociales, ambientales y ahora también relativos al cambio climático ${ }^{3}$ y a la sostenibilidad de las formas de vivir y de producir de las sociedades del siglo XXI.

Dicha metodología se concreta con el denominado modelo LINEX, el cual fue inicialmente aplicado a los casos de algunos países desarrollados como EE. UU., Japón y Alemania (véase el trabajo de Hall et al., 2001). Como resultado general el nuevo factor (E) resultó tener el principal peso en la explicación de la creación de riqueza económica durante la mayor parte del siglo XX. Este logro ha sido reconocido entre otros autores por Rifkin (2011), en cuyo trabajo reconoce que por esta vía se demostró que la mayor parte de los aumentos de productividad y de crecimiento económico registrados en las sociedades industriales se debe a la energía y denominado (E) en dicho modelo.

En función de estos desarrollos metodológicos y también como resultado de sus propias investigaciones Ayres (1978, 1994, 1998, 2001), y Ayres y Warr $(2003,2005,2009)$, dan un paso adicional integrando la segunda ley de la termodinámica para proponer una alternativa al tercer factor productivo denominada ahora exergía $(U)^{4}$, el cual se incorpora en la función de producción Cobb-Douglas junto con los factores clásicos trabajo (L) y el capital $(\mathrm{K})$. La inclusión de este tercer factor permite verificar tanto dicha relación como cuantificar el peso del aporte exergético a la creación de riqueza, esto es, en la formación del PIB. Simultáneamente y como uno de los logros más importantes de esta metodología y contenidos en su trabajo de 2009, se

3 Considerando no sólo la importancia del tema sino la trascendencia de este se sugiere ver Nordhaus (2008).

4 En este punto cabe aclarar que esta nueva variable (E) puede incluir los aportes energéticos explicados y traducidos a base exergética de vectores como el carbón, el petróleo, el gas natural $\mathrm{y}$, en general, todos los recursos primarios que intervienen en los procesos productivos e industriales, aportando su componente efectiva a los procesos productivos y sociales, y puede ser entendida como un indicador de eficiencia termodinámica y de aporte efectivo de trabajo incorporado. 
tiene que con la inclusión de este tercer factor se elimina el llamado residuo de Solow — lo que en otros términos implica endogenizar la tecnología— lo que resulta consistente con los resultados de los trabajos mencionados de Kümmel y demás autores asociados.

En el mismo sentido, aunque con diferentes enfoques se pueden reseñar los trabajos de Schurr et al. (1990), Stern (1993), Beaudreau (1995, 1998, 2005), Pokrovski (2003, 2007), Alam (2009), entre otros, los cuales estudian y discuten tanto la existencia e importancia de dicha relación como sus consecuencias sobre el crecimiento económico, el funcionamiento del sistema económico y sus impactos sobre el entorno natural. En efecto, la relación entre el consumo de energía, el crecimiento económico y el medio ambiente ha recibido una creciente atención en los últimos años. Los resultados presentados en trabajos como los de Esso y Keho (2016), Nahman y Antrobus (2005), Ricci (2007), y Brock y Taylor (2010), son de interés pues, aunque varían los países bajo análisis, tipo de variables asociadas a la producción y al consumo de energía y el tipo de técnicas estadísticas utilizadas para el modelamiento, muestran que en países en vía de desarrollo el nivel de contaminación aumenta hasta que los ingresos exceden un nivel umbral evidencia de la denominada curva ambiental de Kuznets (EKC).

Ahora bien, de acuerdo con Hall y Klitgaard (2012) al analizar el comportamiento económico y productivo de las sociedades modernas debería ser de común aceptación que la energía es un factor determinante para todos los procesos productivos y sociales y que resulta ser tanto o más importante que el capital o el trabajo. En ese sentido, Kümmel (2011) afirma que sin los volúmenes de energía que demandamos diariamente se necesitaría una economía esclavista de dimensiones absurdas con el fin de proporcionar a los ciudadanos los bienes y servicios a los que se han acostumbrado. Por esta razón, desde sus primeros trabajos, este autor sostiene que la energía es un tercer factor productivo, el cual puede introducirse en la función de producción para cuantificar su aporte al PIB.

Esta aproximación a la realidad es consistente con lo que Smil (2005) denomina 'sociedades energético-intensivas', en las cuales se verifica que, desde que se tienen registros, el nivel de ingreso per cápita es directamente 
Manrique-Díaz y Lemus-Polanía: Procedimiento de optimización no lineal...

proporcional al nivel de consumo de energía per cápita ${ }^{5}$. Así, a pesar del creciente número de investigaciones donde se demuestra la importancia económica relativa de la energía para la generación de riqueza, su naturaleza como factor de producción no es considerada o no puede ser entendida ${ }^{6}$ en todas sus dimensiones por el análisis económico neoclásico ${ }^{7}$.

En la realidad, la estructura y el funcionamiento del aparato productivo y del sistema económico de los países industrializados (y de manera lógica también de los países en vías de desarrollo) resultan estar determinados por la necesidad de acceso permanente, eficiente y económico a diferentes vectores energéticos como son el petróleo, el carbón o el gas natural, entre otros. Esta necesidad constituye uno de los logros más importantes de las economías de mercado y es denominada seguridad energética, la cual es fundamentalmente responsabilidad del Estado.

Según Kümmel (1980, 1982), Kümmel et al. (1985) y Lindenberger et al. (2017), dichos vectores energéticos se convierten en un factor de producción en el momento exacto de su liberación de los portadores de energía, es decir, cuando el carbón es utilizado para la producción de energía eléctrica en las centrales termoeléctricas o cuando se quema en los altos hornos para la producción de acero, hierro o cemento, para citar solo algunos ejemplos específicos.

En este contexto, el modelo LINEX —entendido como una variante del clásico modelo Cobb-Douglas de la teoría estándar- desarrollado

5 Una buena aproximación histórica a esta afirmación la proporcionan los trabajos de Humphrey y Stanislav (1979) y Fouquet y Pearson (1988) en sus trabajos de la evolución económica y energética del primer país industrializado del mundo: Inglaterra.

6 Una posible vía para entender el por qué la teoría ortodoxa no se atiene al análisis termodinámico y en particular a la consecuencia que tiene para la economía la ley de la entropía, es que dicha teoría quedó atrapada en el marco y en los límites mecánicos del paradigma newtoniano; en otros términos, esto implica quedar delimitada en lo fundamental por la primera ley de la termodinámica, esto es, la ley general de la conservación de la energía.

7 Desde esta perspectiva analítica, existe un número creciente de trabajos, que se han derivado del seminal de Kraft y Kraft (1978). Sin embargo, sus fundamentos que son básicamente estadísticos han hecho carrera en muchos economistas, sin que exista claridad respecto al concepto de energía y a sus implicaciones en el proceso económico. Con todo, son ampliamente referenciados para la definición de políticas energéticas en numerosos países. 
conceptual y empíricamente por R. Kümmel y los autores que han trabajado con él, y empleado rigurosamente por Ayres y Warr (2005, 2009), es el método más claro y funcional para realizar dicha aproximación. En consecuencia, este modelo es tomado como referente básico para su validación e implementación en el caso de Colombia. Lo anterior, con el objetivo inicial de cuantificar el aporte de la variable energética y su componente exergética en el proceso de crecimiento económico y para verificar su potencialidad explicativa, en comparación con los factores tradicionales de la función de producción neoclásica. A partir de esta verificación se busca explicar de manera alternativa e integral la estructura y evolución del PIB, es decir, del proceso de creación de riqueza, pero esta vez para el caso de una economía en vías de desarrollo.

Así, el principal objetivo de este ejercicio es constatar que los avances conceptuales y empíricos logrados con el modelo LINEX pueden ser extendidos y verificados para el caso de una economía de tamaño medio como es el caso de Colombia. Superando algunas de las dificultades de información existentes ${ }^{8}$, fue posible calcular una serie de energía (exergía) ${ }^{9}$ eléctrica, a partir de la cual se busca verificar su validez estadística, y calcular su peso en la determinación del PIB para Colombia durante el periodo de tiempo analizado ${ }^{10}$. Lo anterior se realizó en función de rigurosos

8 Se debe precisar qué, dadas las limitaciones para obtener información confiable y de largo plazo en relación con la producción y el consumo de vectores energéticos como el carbón, el petróleo, el gas natural y la madera entre otros, el factor exergético que se usa para este trabajo solo incluye el componente de energía eléctrica, a partir de la construcción de una serie de datos para el periodo 1925- 1997.

9 En este caso, se habla de energía eléctrica como exergía en la medida que, como explican Ayres y Warr (2009), este vector energético es exergía pura cuando se emplea en los procesos productivos y de transformación económica. Técnicamente, lo anterior significa que la eficiencia tanto en su producción como en su uso es muy alta.

10 En este punto es importante aclarar que, como consecuencia de la inexistencia de bases de datos energéticos y exergéticos en el país, fue necesario considerar información básica de la Unidad de Planeamiento Minero Energético (UPME) y procesarla junto con variadas fuentes para obtener, inicialmente, la serie de exergía eléctrica empleada por primera vez en este trabajo. Sin embargo, la misma debe ser entendida como uno de los componentes de la serie de exergía total para la economía colombiana que debe incluir los aportes de los diferentes vectores energéticos, como el carbón, el petróleo, el gas natural, la madera, etc. 
Manrique-Díaz y Lemus-Polanía: Procedimiento de optimización no lineal...

análisis estadísticos previos que incluyen el uso del algoritmo de LevenbergMarquardt el cual permite resolver un problema de mínimos cuadrados no lineales con condiciones de frontera no lineales y expresadas en forma de desigualdades en función del cual fue posible validar tanto el modelo propuesto como sus presupuestos teóricos y conceptuales.

El cuerpo de este trabajo se desarrolla en tres partes: en la primera se tratan y explicitan los factores de producción y su proceso de agregación; en la segunda se introduce el factor (E) tanto en la función clásica CobbDouglas, como en la función LINEX. Finalmente, en la tercera parte se discuten los resultados del ejercicio y se consignan las conclusiones para el caso de Colombia.

\section{Los factores de producción y su agregación}

Las dos funciones de producción consideradas en este trabajo están sujetas a los dos elementos básicos del proceso de producción industrial: la ejecución de trabajo y el procesamiento de información. De esta observación se obtienen los principios de agregación que permiten resumir a nivel macroeconómico el conjunto heterogéneo de entradas y salidas de cada función. En resumen, y concordancia con el enfoque analítico tradicional, las variables empleadas $\operatorname{son}^{11}$ :

- El producto interno bruto (PIB) corregido por la inflación (Y) (Grupo de Estudios del Crecimiento Económico —GRECO_, 2004, p. 21).

- El capital real agregado disponible corregido por la inflación (K). Comprende los desembolsos en concepto de adiciones a los activos fijos de la economía más las variaciones netas en el nivel de los inventarios. Variable medida y agregada en millones de pesos colombianos (GRECO, 2004).

11 Cabe señalar que el PIB corregido por la inflación (Y), el capital real agregado disponible corregido por la inflación $(\mathrm{K})$ y la población económicamente activa $(\mathrm{L})$ fueron obtenidas de las series oficiales procesadas por el Grupo de Estudios del Crecimiento Económico del Banco de la República (GRECO, 2004), y la serie del consumo de energía eléctrica en base exergética (E) fue producida por una parte del equipo de investigación entre 2012 y 2013. 
- La población económicamente activa (L). Esta variable se define como las personas en edad de trabajar, que trabajan o están buscando empleo. En el presente estudio se usa el total de personas en edad de trabajar que trabajan como proxy de esta población (GRECO, 2004).

- El consumo final de energía eléctrica (E) expresada en base exergética, el cual se presenta de manera agregada y medida en terajoules-año (TJ/año).

Resulta importante definir la siguiente notación:

- $\alpha$ denota la elasticidad del PIB al capital.

- $\beta$ denota la elasticidad del PIB a la población económicamente activa.

- $\gamma$ denota la elasticidad del PIB a la energía.

\section{Funciones de producción consideradas}

En esta sección del documento se presentan las diferentes funciones de producción consideradas.

\section{A. Exergía en el modelo de crecimiento de Cobb-Douglas}

En el modelo tradicional de Cobb-Douglas, los factores de producción asociados al capital, la población económicamente activa y la exergía $\left(K_{t}, L_{t}\right.$, $\left.E_{t}\right)$ son considerados como variables independientes (Ayres \& Voudouris, 2014). La suposición es que alguna combinación de estas variables puede explicar los cambios en el PIB $\left(Y_{t}\right)$ de Colombia durante un largo período de tiempo. El modelo considerado es el siguiente

$$
Y_{t}=A_{t} K_{t}^{\alpha} L_{t}^{\beta} E_{t}^{\gamma}
$$

donde $A_{t}$ es una medida de innovación usualmente denominada "progreso tecnológico". La condición de rendimientos constantes implica que

$$
\alpha+\beta+\gamma=1
$$


Manrique-Díaz y Lemus-Polanía: Procedimiento de optimización no lineal...

En la Figura 1 se puede observar que el crecimiento en el consumo de energía eléctrica en el país ha aumentado de manera acelerada a partir de los años 60, a una tasa que sobrepasa considerablemente la obtenida para los demás factores considerados. Nótese que dicho crecimiento es tan acelerado que oculta el crecimiento de los demás factores considerados en el estudio. Con el fin de que el lector pueda observar el crecimiento del PIB, el capital y la PEA se presenta, en la Figura 2, el índice (1925:1) de los factores de producción en Colombia incluyendo y excluyendo el consumo de energía eléctrica durante el periodo de tiempo considerado.

Figura 1. Índice 1925:1 del PIB y los factores de producción (Colombia, 1925-1997)

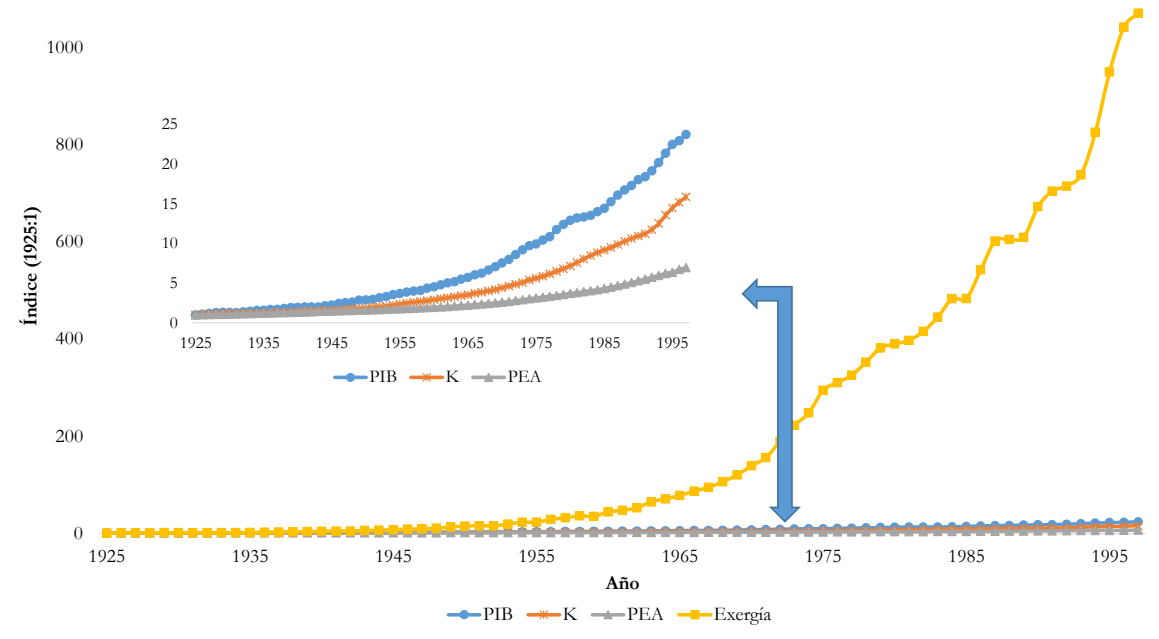

Fuente: elaboración propia.

Según Kümmel et al. (1985) y Ayres y Warr (2005, 2009) las soluciones más simples (triviales) para la condición de rendimientos constantes establecidas en la ecuación (2) son valores numéricos de la forma $\alpha=\alpha_{0}$, $\beta=\beta_{0}$ y $\gamma_{0}=1-\alpha_{0}-\beta_{0}$. Para el caso de una economía de dos factores, se obtiene la función Cobb-Douglas original, donde $\gamma_{0}=0$. En el caso colombiano se toman como referencia los resultados presentados por el GRECO del Banco de la República, en los que se puede observar que la participación promedio durante el periodo 1926-1994 fue de 0,38225 y 0,61775 para $\alpha_{0}$ y $\beta_{0}$, respectivamente (véase GRECO, 2004, p. 56). 
Figura 2. Índice 1925:1 del PIB real y los factores de producción sin incluir el crecimiento del consumo de energía eléctrica (Colombia, 1925-1997)

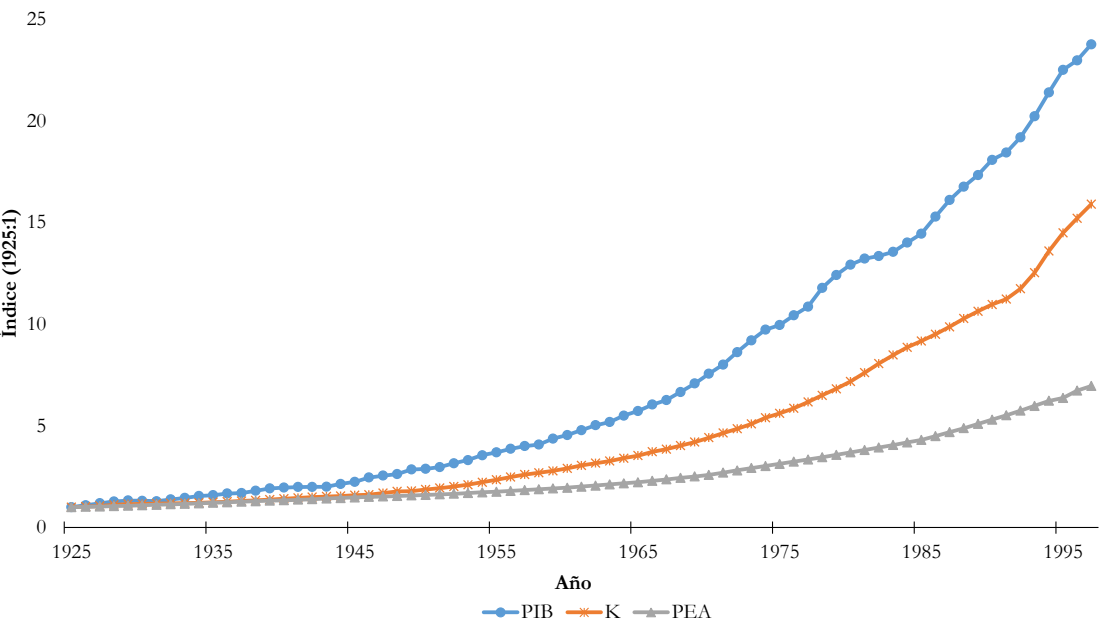

Fuente: elaboración propia.

La Figura 3 presenta claramente que la función Cobb-Douglas — sin considerar la exergía como tercera variable independiente, pero manteniendo la condición de rendimientos constantes y con elasticidades proporcionales a la participación en los pagos a los recursos de entrada en las cuentas nacionales - no explica el crecimiento histórico de Colombia en el largo plazo, pues se puede observar que los residuales del modelo ajustado crecen de forma exponencial.

Volviendo al modelo estándar de Solow, y los supuestos que le acompañan, el factor $A_{t}$ se ajustó de forma independiente al residuo no explicado que una vez fue llamado "progreso tecnológico" o, más recientemente, la productividad total de los factores (PTF). En el presente trabajo se determinó que el "mejor ajuste" para la función de progreso técnico en Colombia durante todo el período 1925-1997 (que se muestra en la Figura 4) está dado por la siguiente expresión:

$$
A_{t}=\exp [0,038143(t-1925)]-1
$$

donde $t$ es el año. 
Manrique-Díaz y Lemus-Polanía: Procedimiento de optimización no lineal...

Figura 3. PIB vs ajuste modelo Cobb-Douglas con elasticidades fijas L(0,61775),

$$
K(0,38225) \text { y } E(0)
$$

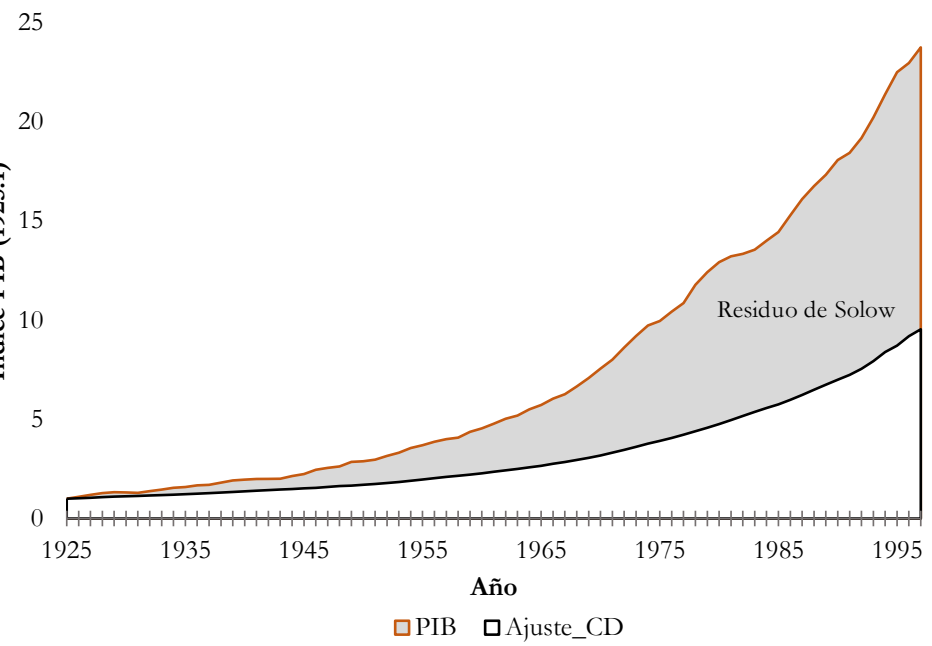

Fuente: elaboración propia.

Figura 4. Residuos de Solow y la minimización de la brecha asociada al progreso tecnológico

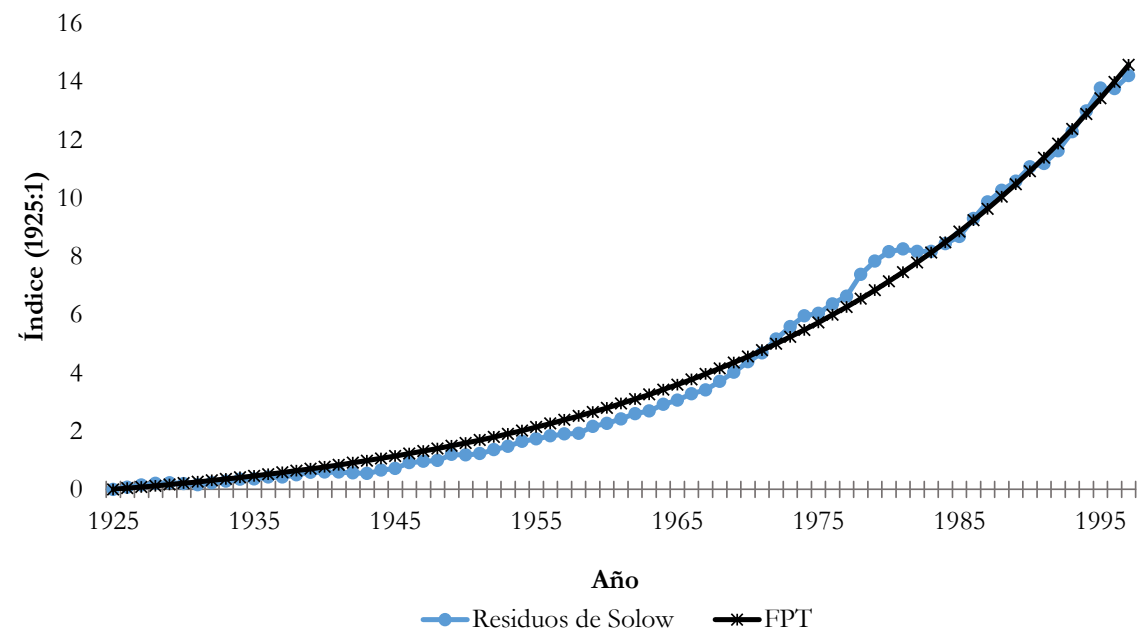

Fuente: elaboración propia. 


\section{B. Exergía en la función de producción LINEX}

Según Kümmel (1980, 1982) y Kümmel et al. (1985), las expresiones matemáticas para las elasticidades de producción $\alpha, \beta$ y $\gamma$, basándose en condiciones de límite asintóticas, deben satisfacer la condición de Euler y ser funciones homogéneas de orden cero de las variables independientes. Siguiendo la notación propuesta en Kümmel et al. (2002) todos los factores de producción considerados están en unidades normalizadas a un año base. Considerando 1925 como periodo de referencia, se tiene que $y_{t}=Y_{t} / Y_{1925}$, $e_{t}=E_{t} / E_{1925}, l_{t}=L_{t} / L_{1925}$ y $k_{t}=K_{t} / K_{1925}$.

La primera de las soluciones, propuestas por las referencias mencionadas previamente, se establece como una forma de la ley de rendimientos decrecientes de capital. Se trata de una condición límite asintótica que trae la noción de que incluso en un estado futuro hipotético de uso intensivo de capital, en la que todos los productos son producidos por máquinas, se mantendrá alguna necesidad irreductible de trabajo L y exergía E, la cual expresan de la siguiente manera:

$$
\alpha_{t}=a_{t}\left(\frac{l_{t}+e_{t}}{k_{t}}\right) .
$$

La segunda ecuación refleja la continua sustitución de trabajo por capital y exergía a medida que la intensidad de capital aumenta (automatización):

$$
\beta_{t}=a_{t}\left(c_{t} \frac{l_{t}}{e_{t}}-\frac{l_{t}}{k_{t}}\right) \text {. }
$$

El supuesto de rendimientos constantes a escala implica que, en cada momento en el tiempo,

$$
\gamma_{t}=1-\alpha_{t}-\beta_{t}
$$

que es la condición de retornos constantes. La integración parcial de la ecuación de crecimiento produce la función llamada LINEX (linealexponencial):

$$
y_{t}=y_{0} e_{t} \exp \left[a_{t}\left(2-\left(\frac{l_{t}+e_{t}}{k_{t}}\right)\right)+a_{t} c_{t}\left(\frac{l_{t}}{e_{t}}-1\right)\right],
$$


Manrique-Díaz y Lemus-Polanía: Procedimiento de optimización no lineal...

donde $y_{0}$ es una constante de integración que mide la valoración monetaria promedio de la canasta original de bienes y servicios que componen la unidad de salida. Las funciones del tiempo $a_{t}$ y $c_{t}$ han sido caracterizadas por Kümmel (1980, 1982) y Kümmel et al. (1985) como "la eficiencia del capital" y "la demanda de energía", respectivamente. Lo que resulta interesante son las productividades dependientes del tiempo que muestran un aumento significativo en la productividad exergética y la disminución de la productividad del trabajo en el tiempo en Colombia ${ }^{12}$.

Al comparar las funciones de producción para el PIB, la función CobbDouglas de la ecuación (1) y la función de LINEX en la (6), se puede obtener una expresión para el multiplicador $A_{t}$ en la ecuación (1), en términos de $K$, $L$ y $E$ :

$$
A_{t}=k_{t}^{-\alpha_{o}} l_{t}^{-\beta_{0}} e_{t}^{\alpha_{0}+\beta_{0}} \exp \left[a_{t}\left(2-\left(\frac{l_{t}+e_{t}}{k_{t}}\right)\right)+a_{t} c_{t}\left(\frac{l_{t}}{e_{t}}-1\right)\right],
$$

siendo $\alpha_{0}$ y $\beta_{0}$ la participación promedio del capital y el trabajo durante el periodo 1926-1994, respectivamente (véase GRECO, 2004, p. 56). En la ecuación (7) se puede observar que $A(t)$ es una expresión que depende fuertemente de E y débilmente (e inversamente) de K y L. Por lo tanto, si el modelo en (6) se ajusta razonablemente a los datos del PIB de Colombia, $A_{t}$ puede ser considerado como una función de la eficiencia de los recursos de conversión empleados en el proceso productivo.

\section{Resultados}

Los datos utilizados en este estudio corresponden a observaciones anuales del PIB corregido por la inflación $(\mathrm{Y})^{13}$, el capital real agregado disponible $(\mathrm{K})^{14}$, la población económicamente activa $(\mathrm{L})$ expresada a través

12 Se empleó el método de optimización no lineal de Levenberg-Marquardt, bajo el supuesto de elasticidades de producción no negativas, para ajustar la función de producción basada en el modelo LINEX.

13 El PIB fue medido y agregado en miles de millones de pesos colombianos.

$14 \mathrm{Al}$ igual que el PIB, el capital real fue medido y agregado en miles de millones de pesos colombianos 
del número de personas empleadas al año y el consumo final de energía eléctrica (E) expresada en base exergética; agregada y medida en terajoules por año (TJ/año). Los datos del PIB, el capital real agregado disponible (K) y la población económicamente activa (L) se obtuvieron de los resultados reportados por el grupo de investigación del Banco de la República (GRECO, 2004), mientras que los datos del consumo final de energía eléctrica (E) en base exergética fueron producidos por una parte del equipo de investigación entre 2012 y 2013. La periodicidad de las series temporales es anual y se consideraron los registros de 1925 a 1997.

\section{A. Modelo de crecimiento estándar: Cobb-Douglas}

En esta subsección se considera el procedimiento presentado en Ayres y Warr (2005, 2009), el cual consiste en ajustar el modelo Cobb-Douglas para las series de tiempo consideradas en este estudio, la productividad total de los factores y la restricción de que la suma de las elasticidades es igual a uno. El modelo de regresión por ajustar se obtiene al aplicar logaritmo en ambos lados de la igualdad en la ecuación (1):

$$
\log \left(Y_{t}\right)=\log \left(A_{t}\right)+\alpha \log \left(K_{t}\right)+\beta \log \left(L_{t}\right)+\gamma \log \left(E_{t}\right)+\varepsilon .
$$

Finalmente, bajo la condición de rendimientos constantes $(\gamma=1-\alpha-\beta)$, se obtiene lo siguiente:

$$
\log \left(Y_{t}\right)=\log \left(A_{t}\right)+\alpha \log \left(K_{t}\right)+\beta \log \left(L_{t}\right)+(1-\alpha-\beta) \log \left(E_{t}\right)+\varepsilon .
$$

En la ecuación anterior, $A_{t}$ es la medida de innovación denominada "progreso tecnológico" y que se presenta en la Ecuación (1), y $\varepsilon$ denota el término de error en el ajuste del modelo Cobb-Douglas. Despejando $\log \left(E_{t}\right)$ y agrupando términos semejantes en (8) se obtiene el siguiente modelo de regresión:

$$
\begin{array}{r}
\log \left(Y_{t}\right)-\log \left(E_{t}\right)=\log \left(A_{t}\right)+\alpha\left(\log \left(K_{t}\right)-\log \left(E_{t}\right)\right)+ \\
\beta\left(\log \left(L_{t}\right)-\log \left(E_{t}\right)\right)+\varepsilon .
\end{array}
$$

Por lo tanto, para garantizar la condición de rendimientos constantes se debe ajustar el modelo de regresión lineal múltiple (MRLM), empleando como 
Manrique-Díaz y Lemus-Polanía: Procedimiento de optimización no lineal...

método de optimización la minimización de la suma de cuadrados del error (SSE) (véase Ayres \& Warr, 2005). En este modelo, la variable respuesta será $\log \left(Y_{t}\right)-\log \left(E_{t}\right)$, denotada $\log Y E$, y sus covariables serán $\log \left(K_{t}\right)-\log \left(E_{t}\right)$, denotada $\log K E$, y $\log \left(L_{t}\right)-\log \left(E_{t}\right)$, denotada $\log L E$. Los resultados obtenidos para el caso colombiano se presentan en la Tabla 1.

Tabla 1. Pruebas individuales de los coeficientes del modelo de regresión ajustado

\begin{tabular}{lcccc}
\hline & Estimador & Error estándar & Estadístico de prueba & Valor $\mathbf{p}$ \\
\hline Intercepto & 9,7823 & 2,7245 & 3,591 & $0,000613^{* * *}$ \\
$\log \left(A_{t}\right)$ & $-0,0053$ & 0,0013 & $-4,057$ & $0,000129^{* * *}$ \\
$\log K E$ & 0,6317 & 0,0626 & 10,085 & $3,29 \mathrm{e}-15^{* * *}$ \\
$\log L E$ & 0,2171 & 0,0652 & 3,331 & $0,001393^{* *}$ \\
\hline
\end{tabular}

Fuente: elaboración propia.

En la Tabla 1 se puede observar que todas las elasticidades obtenidas por el modelo Cobb-Douglas y sus covariables son estadísticamente significativas, es decir, aportan a la explicación de la variable respuesta (LogPIB). En la Tabla 2 se presentan las elasticidades estimadas y sus respectivos intervalos de confianza del $95 \%$ :

Tabla 2. Estimación puntual y por intervalos de las elasticidades - modelo Cobb-Douglas

\begin{tabular}{lccc}
\hline Parámetro & Valor estimado & Límite inferior & Límite superior \\
\hline$\alpha$ & 0,2214 & 0,0673 & 0,3755 \\
$\beta$ & 0,4858 & 0,3393 & 0,6323 \\
$\gamma=1-\alpha-\beta$ & 0,2927 & 0,0000 & 0,5933 \\
\hline
\end{tabular}

Fuente: elaboración propia.

En la Figura 5 se presenta una comparación entre el PIB real colombiano (COP, miles de millones) y los valores estimados de esta serie empleando el modelo Cobb-Douglas en la ecuación (9). Se puede apreciar que las estimaciones realizadas por el modelo toman valores muy cercanos al PIB real colombiano hasta mediados de los años 70 . A partir de este periodo 
el ajuste del modelo Cobb-Douglas se empobrece considerablemente. Los resultados obtenidos en esta subsección parecen indicar que, bajo el modelo tradicional, el consumo de energía eléctrica (E) en Colombia contribuye de manera significativa en el crecimiento económico del país; esto contradice los resultados tradicionales de una economía basada en dos factores.

Figura 5. Ajuste del modelo Cobb-Douglas al PIB real de Colombia (1925-1997)

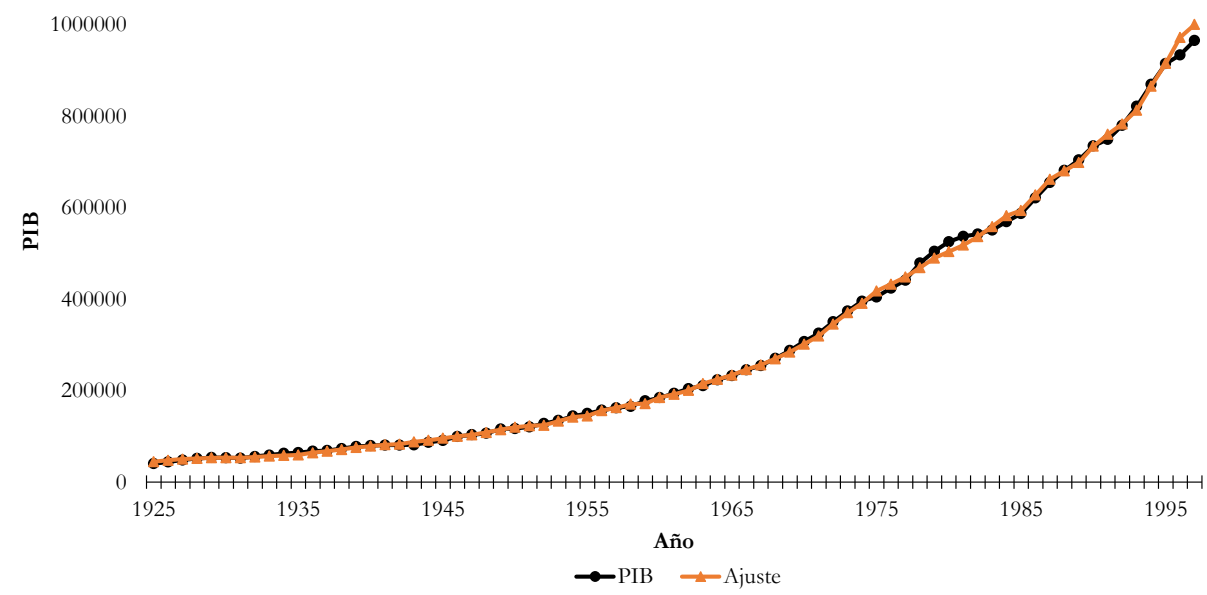

Fuente: elaboración propia.

\section{B. Modelo de crecimiento alternativo: LINEX}

Siguiendo el modelo LINEX presentado en Kümmel et al. (2002), tenemos que

$$
y_{t}=y_{0} e_{t} \exp \left\{a_{t}\left(2-\frac{l_{t}+e_{t}}{k_{t}}\right)+a_{t} c_{t}\left(\frac{l_{t}}{e_{t}}-1\right)\right\},
$$

en donde $y_{t}=Y_{t} / Y_{1925}, e_{t}=E_{t} / E_{1925}, l_{t}=L_{t} / L_{1925}, k_{t}=K_{t} / K_{1925}$ son las variables macroeconómicas indexadas respecto al año base (1925). Los coeficientes $a_{t}$ y $c_{t}$ están dados por:

$$
a_{t}=\frac{a_{1}-a_{2}}{1+\exp \left\{-a_{3}\left(t-a_{4}\right)\right\}}+a_{2} ; a_{1}>a_{2}
$$


Manrique-Díaz y Lemus-Polanía: Procedimiento de optimización no lineal...

$$
c_{t}=\frac{c_{1}-c_{2}}{1+\exp \left\{-c_{3}\left(t-c_{4}\right)\right\}}+c_{2} ; \quad c_{1}<c_{2} .
$$

En las ecuaciones (11) y (12), $a_{1}, \ldots, a_{4}, c_{1}, \ldots, c_{4}$ son parámetros positivos que se estiman minimizando la distancia cuadrática $\sum_{t=1}^{T}\left[\tilde{y}_{t}-y_{t}\right]^{2}$, es decir, la diferencia cuadrática entre el valor estimado del PIB, empleando el modelo LINEX $\left(\tilde{y}_{t}\right)$, y su valor real $\left(y_{t}\right)$. En el proceso de estimación se empleó el método de optimización no lineal de Levenberg-Marquardt, bajo el supuesto de elasticidades de producción no negativas, para ajustar la función de producción basada en el modelo LINEX.

En la Figura 6 se presenta el valor anual de los polinomios $a_{t}$ y $c_{t}$ enunciados en las ecuaciones (11) y (12) y calculados para el caso colombiano durante el periodo de tiempo considerado 1925-1997.

Figura 6. Polinomios a (t) y $c(t)$ del modelo LINEX para Colombia (1925-1997)
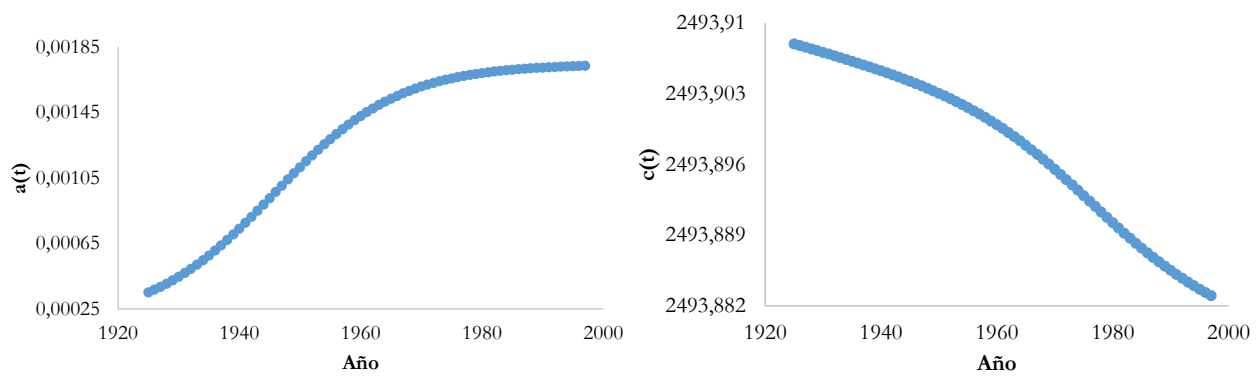

Fuente: elaboración propia.

En la Figura 7 se presenta el valor anual de las productividades marginales de $\alpha, \beta$ y $\gamma$ (elasticidades de producción) para el caso colombiano durante el periodo comprendido entre 1925 y 1997. En este gráfico se puede observar un quiebre estructural en las pendientes de las curvas $\beta$ y $\gamma$ algunos años antes de 1935; este quiebre puede estar asociado a la marcada transición en el modelo 
económico de desarrollo del país ${ }^{15}$ y el rápido ritmo de crecimiento industrial, que se convirtió en un hito inigualable en el resto de América Latina y en la historia posterior del país (véase Aspe, Dornbusch \& Obstfeld, 1983, p. 5-40). Una descripción detallada del panorama económico y político de Colombia durante los años 30 se puede encontrar en Ocampo y Montenegro (1982).

Figura 7. Elasticidades estimadas por el modelo LINEX para Colombia (1925-1997)

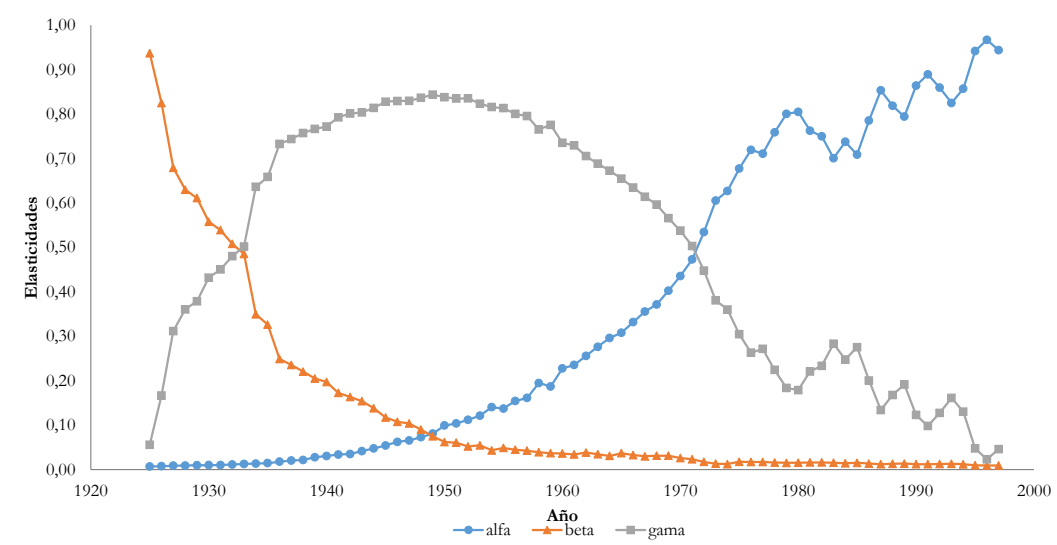

Fuente: elaboración propia.

Las elasticidades promedio obtenidas por el modelo LINEX para Colombia durante el periodo 1925-1997 fueron:

1. $\bar{\alpha}=0,3619$ : promedio asociado al capital real agregado disponible.

2. $\bar{\beta}=0,1359$ : promedio asociado a la población económicamente activa.

3. $\bar{\gamma}=0,5022$ : promedio asociado al consumo de energía eléctrica.

Desde la perspectiva macroeconómica y del equilibrio general, los resultados obtenidos se pueden interpretar en el sentido que el factor de pago asociado a la energía eléctrica (E) en Colombia y registrado en las

15 Durante este periodo se desarrollaron muchos de los instrumentos básicos de intervención del Estado en la economía como, por ejemplo, el control de cambios, el régimen fiduciario organizado en torno al Banco de la República, el impuesto moderno de renta y patrimonio, entre otros. 
Manrique-Díaz y Lemus-Polanía: Procedimiento de optimización no lineal...

cuentas nacionales ha sido mayor que el del capital $(\mathrm{K})$ y la población económicamente activa (L), durante el periodo de tiempo considerado. Sin embargo, los economistas ortodoxos podrían decir que en Colombia los pagos a los factores de producción capital $(\mathrm{K})$, trabajo (L) y energía (E) se desvían dramáticamente de los de los países de la OCDE, o que las elasticidades de producción de este manuscrito deben ser tan erróneas como las obtenidas por Kümmel, Ayres y todos los autores que han contribuido al desarrollo del modelo empleado en este trabajo.

Sin embargo, creemos que nuestros resultados confirman los hallazgos de los autores previamente mencionados, a saber, que la elasticidad de la variable energía es mucho mayor y que la de la mano de obra es mucho menor que los costos compartidos respectivos de estos factores. En consecuencia, los resultados obtenidos permiten validar el modelo empleado en el caso de Colombia y concluir que la condición de rendimientos constantes de la función de producción neoclásica no se aplica a ninguna de las economías que se han estudiado hasta ahora con esta innovadora metodología.

Finalmente, en la Figura 8 se presentan los valores ajustados por el modelo LINEX en comparación con los datos reales del PIB. Se puede apreciar que las estimaciones realizadas por el modelo toman valores muy cercanos al PIB real colombiano hasta inicios de los años 90. A partir de este año el ajuste del modelo LINEX se empobrece considerablemente.

Figura 8. Ajuste del modelo LINEX a la serie del PIB de Colombia (1925-1997)

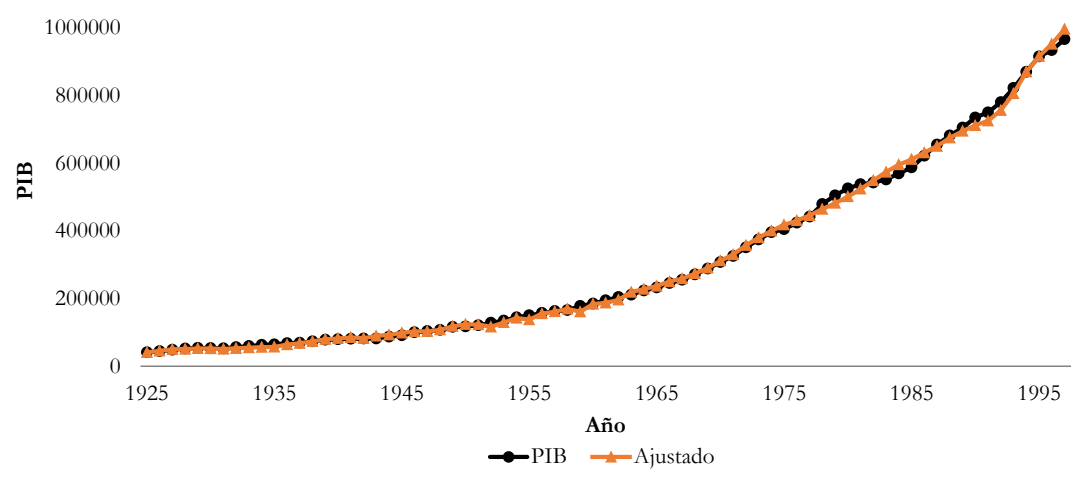

Fuente: elaboración propia. 


\section{Conclusiones}

Si bien las investigaciones para formalizar una conexión entre la economía y la energía llevan varias décadas, ahora tenemos la posibilidad de relacionar la teoría del crecimiento económico con la variable energética con importantes resultados, independientemente del método empleado. En general, los análisis existentes revelan un creciente interés por establecer relaciones, formular metodologías y realizar rigurosos ejercicios para comprender, cuantificar y analizar las implicaciones de la producción y uso de la energía en las economías modernas. Desde la perspectiva heterodoxa con la inclusión de la energía como un tercer factor en la función de producción estándar se están logrado importantes resultados como revela el análisis realizado para el caso de Colombia una vez aplicado el modelo LINEX al análisis del crecimiento en el periodo de estudio.

En efecto, de una parte los resultados encontrados para Colombia permiten verificar que aunque en el modelo Cobb-Douglas ajustado todas las elasticidades obtenidas y sus variables regresoras asociadas son estadísticamente significativas; es decir, aportan en la explicación de la variable respuesta ( $\log \mathrm{PIB})$, al realizar la verificación de los supuestos del modelo se determinó que ninguno se cumple, lo cual implica que los resultados obtenidos por el mismo están considerablemente sesgados y no reproducen la verdadera relación entre el PIB con el capital, la población económicamente activa y el consumo de energía eléctrica.

En el caso del modelo alternativo LINEX (véase Figura 7), se encuentra que los resultados con los factores de producción considerados tienen un comportamiento concordante con lo presupuestado en dicho modelo, demostrando al menos tres hechos fundamentales: primero, que la participación de la energía eléctrica fue la fuente más importante de creación del PIB nacional en el periodo analizado. Segundo, que el aporte del capital fue ganando importancia hasta convertirse en el principal factor de creación del PIB, especialmente desde mediados de los años 70 del siglo XX. Y tercero, que la participación de la fuerza laboral en la composición del PIB disminuyó, especialmente desde los años 40, hasta aparecer marginalmente al final del periodo analizado. Estos resultados son interesantemente similares a 
Manrique-Díaz y Lemus-Polanía: Procedimiento de optimización no lineal...

los obtenidos por Lindenberger et al. (2017) para la economía estadounidense y alemana durante un periodo de tiempo muy similar al estudiado para el país.

En consecuencia, es claro que existe una metodología alternativa que permite explicar el crecimiento económico en el país, con arreglo a un modelo que, si bien no es conocido en nuestro medio, sí resulta potencialmente poderoso para explicar el proceso de creación de riqueza material desde una perspectiva de investigaciones de frontera. Estas investigaciones combinan el conocimiento de diferentes disciplinas y, además, permiten dinamizar el análisis del crecimiento económico en la medida que posibilitan ver la evolución en el tiempo y la participación de las elasticidades asociadas con las variables estándar $(\mathrm{K})$ y $(\mathrm{L})$ y la variable nueva $(\mathrm{E})$, es decir, para nuestro análisis la energía (exergía) eléctrica.

Con todo, parece necesario profundizar en porqué la participación de la energía eléctrica (E) en la creación de riqueza fue más importante desde inicios del periodo y hasta los años setenta del siglo XX y en por qué disminuye desde entonces hasta el fin del periodo analizado. En principio, este resultado sugiere que si bien durante el primer subperiodo la energía eléctrica (E) resultaba determinante de la creación de riqueza (Kalmanovitz, 2010, p. 132), con la rápida urbanización del país, impulsada desde mitad del siglo XX, y el menor peso específico del aparato industrial en la creación y composición del PIB, dicha participación en la creación de riqueza empezó a decrecer también como consecuencia de la mejora en la eficiencia de los aparatos empleados por las industrias y los consumidores en general, la cual ha ido aumentando, justamente desde los años 80 del pasado siglo XX.

También resulta interesante observar cómo desde los años setenta el capital $(\mathrm{K})$, empezó a repuntar como el principal factor de producción en la economía colombiana, lo cual refuerza la tesis de la urbanización del país y de la modernización al menos de algunos sectores productivos representativos.

Aunque no son concluyentes, dichos resultados representan un efecto combinado sobre la economía colombiana y derivado del impacto negativo de la llamada crisis energética; los esfuerzos para modernizar la base industrial y productiva del país, factores que explican, al menos parcialmente, el cambio de tendencia en la participación del capital y la energía. 
Sin embargo, esta situación resulta paradójica en el sentido de que durante estos años el país realizó grandes inversiones en infraestructura eléctrica, en un proceso que permitió la construcción de un importante número de proyectos de generación de energía eléctrica e inversiones intensivas en capital. Dichas inversiones fueron realizadas en un contexto donde al tiempo que la industria se estaba debilitando, la urbanización del país se estaba consolidando.

Con todo, será necesario profundizar y ahondar en este análisis del papel de la energía en el proceso productivo, para lo cual será necesario ampliar tanto la base de información relativa a la contribución energética y exergética, así como en las demás variables las cuales deben ser obtenidas y validadas estadísticamente en diferentes intervalos de tiempo.

Finalmente, cabe decir que si bien el trabajo de Kümmel y los autores relacionados con esta metodología han llevado el debate relativo a los determinantes del crecimiento económico a un punto muy interesante en relación con el paradigma dominante, sus proyecciones apuntan a analizar el resultado de esta realidad en función de fenómenos tan complejos como el cambio climático y la sostenibilidad del desarrollo económico y social, en la medida que estos procesos dependen hasta ahora de los recursos que más contaminan la atmosfera. Será necesario entonces lograr mayores niveles de eficiencia, tanto en la producción como en el consumo de energía, si se quiere lograr mantener los actuales estándares de vida sin afectar de manera irreversible el medio natural en el que vivirán las generaciones futuras.

\section{Agradecimientos}

Este artículo es un resultado del proyecto: "Energía y crecimiento económico en Colombia: un análisis integral e interdisciplinario", financiado por el Ministerio de Ciencia, Tecnología e Innovación, MinCiencias, Ref.: 111852129363. 
Manrique-Díaz y Lemus-Polanía: Procedimiento de optimización no lineal...

\section{Referencias}

Akarka, A. \& Long, T.-V. (1980). On the relationship between energy and GNP: A reexamination, Journal of Energy and Development, 5(2), 326-331.

Alam, S. (2009). Bringing energy back into the economy: examining economic growth with energy, Review of Radical Political Economics, 41(2), 170-185.

Allen, E. L. (1979). Energy and Economic Growth in the United States, Massachusetts: The MIT Press

Altinay, G. \& Karagol, E. (2004). Structural break, unit root, and the casuality between energy consumption and GDP in Turkey, Energy Economics, 26(6), 985-994.

Apergis, N. \& Payne, J. (2010a). Coal consumption and economic growth: Evidence from a panel of OECD countries, Energy Policy, 38(3), 1353-1359.

Apergis, N. \& Payne, J. (2010b). Energy consumption and growth in South America: Evidence from a panel error correction model, Energy Economics, 32(6), 1421-1426.

Asafu-Adjaye, J. (2000). The relationship between energy consumption, energy prices and economic growth: time series evidence from Asian developing countries, Energy Economics, 22(6), 615-625.

Aspe, P., Dornbusch, R. \& Obstfeld, M. (1983). Financial Policies and the World Capital Market: The Problem of Latin American Countries, Chicago: University of Chicago Press.

Ayres, R. (1978). Application of Physical Principles to Economics, Resources, Environment and Economics: Applications of the Materials/Energy Balance Principle. New York: Jhon Wiley y Sons.

Ayres, R. (1994). Information, Entropy, and Progress: A New Evolutionary Paradigm. New York: AIP Press. 
Ayres, R. (1998). Technological progress: a proposed measure, Technological Forecasting and Social Change, 59(3), 213-233.

Ayres, R. (2001). The minimum complexity of endogenous growth: the role of physical resource flows, Energy - The International Journal, 26(9), 817-838.

Ayres, R. \& Ayres, E. (2010). Crossing the Energy Divide: Moving from Fossil Fuel Dependence to a Clean-Energy Future, New Jersey: Prentice Hall

Ayres, R. \& Warr, B. (2003). Exergy, power and work in the US economy 1900-1998, Energy - The International Journal, 28(3), 219-273.

Ayres, R. \& Warr, B. (2005). Accounting for growth: the role of physical work, Structural Change and Economic Dynamics, 16(2), 181-209

Ayres, R. \& Warr, B. (2009). The Economic Growth Engine: How energy and work drive material prosperity, Northampton-Massachusetts: Edward Elgar Publishing, Inc.

Ayres, R. \& Voudouris, V. (2014), The economic growth enigma: Capital, labor, and useful energy?, Energy Policy, 64(1), 16-28.

Barreto, C. A. \& Campo R., J. (2012). Relación a largo plazo entre consumo de energía y PIB en América Latina: Una evaluación empírica con datos panel. Ecos de Economía, 16(35), 73-89.

Beaudreau, B. (1995). The impact of electric power in productivity: A study of US manufacturing 1950-1984, Energy Economics, 17(3), 231-236.

Beaudreau, B. (1998). Energy and Organization: Growth and Distribution Reexamined, Santa Barbara: Greenwood Press.

Beaudreau, B. (2005). Engineering and economic growth, Structural Change and Economic Dynamics, $16(2), 211-220$.

Belke, A., Dobnik, F. \& Dreger, C. (2011). Energy consumption and economic growth: new insights into the cointegration relationship, Energy Economics, 33(5), 782-789. 
Manrique-Díaz y Lemus-Polanía: Procedimiento de optimización no lineal...

Belloumi, M. (2009). Energy consumption and GDP in Tunisia: Cointegration and causality analysis, Energy Policy, 37(7), 2745-2753.

Berndt, E. R. \& Wood, D. O. (1975). Technology, Prices, and the Derived Demand for Energy. The Review of Economics and Statistics, 17(3), 259-268.

Bildirici, M. \& Kayikci, F. (2012). Economic growth and electricity consumption in former Soviet Republics, Energy Economics, 34(3), 747-753.

Brock, W. \& Taylor, S. (2010). The green Solow model. Journal of Economic Growth, 15(2), 127-153.

Campo R. J. \& Sarmiento, V. (2011). Un modelo de corrección de errores para la relación entre el consumo de energía y el PIB en Colombia (19702009). Revista Finanzas y Política Económica, 3(1), 59-70.

Cheng, B. (1997). Energy consumption and Economic Growth in Brazil, Mexico and Venezuela: A Time Series Analysis, Applied Economics Letters, 4(11), 671-674.

Chiou-Wei, S-Z., Chen, C.-F. \& Zhu, Z. (2008). Economic growth and energy consumption revisited - Evidence from linear and nonlinear Granger causality, Energy Economics, 30(6), 3063-3076.

Chontanawat, J., Hunt, L. \& Pierse, R. (2008). Does energy consumption cause economic growth? Evidence from a systematic study over 100 countries, Journal of Policy Modeling, 30(2), 209-220.

Cobb, G. W., and Douglas, P.N. (1928). A theory of production. American Economic Review, 18(1), 139-165.

Cottrell, F. (1955). Energy and Society. New York: McGraw Book Company.

Ebohon, O.-J. (1996). Energy, Economic Growth and Causality in Developing Countries: A Case Study of Tanzania and Nigeria, Energy Policy, 24(5), 447-453. 
Esso, L. \& Keho, Y. (2016). Energy consumption, economic growth, and carbon emissions: Cointegration and causality evidence from selected African countries, Energy, 114(2), 492-497

Farhani, S. \& Ben-Rejeb, J. (2012). Link between Economic Growth and Energy Consumption in Over 90 Countries, Interdisciplinary Journal of Contemporary Research in Business, 3(11), 282-297.

Fouquet, R., and Pearson, J. G. (1998). A Thousand Years of Energy Use in the United Kingdom. The Energy Journal, 19(4), 1-41.

Georgescu-Roegen, N. (1971). The Entropy Law and the Economic Process. Cambridge: Harvard University Press.

Georgescu-Roegen, N. (1975). Energy and Economic Myths, Southern Economic Journal, 41(3), 347-381.

Georgescu-Roegen, N. (1977). The Steady State and Ecological Salvation: A Thermodynamic Analysis, BioScience, 27(4), 266-270.

GRECO (Grupo de Estudios del Crecimiento Económico), GRECO (2004), El Crecimiento Económico Colombiano en el siglo XX, Bogotá: Banco de la República.

Gurgul, H. \& Lach, L. (2012). The electricity consumption versus economic growth of the Polish economy, Energy Economics, 34(2), 500-510.

Hall, C. \& Klitgaard, K. (2012). Energy and the Wealth of Nations: Understanding the Biophysical Economy, New York: Springer

Hall, C., Lindenberger, D., Kümmel, R., Kroeger, T. \& Eichhorn, W. (2001). The Need to Reintegrate the Natural Sciences with Economics, BioScience, 51(8), 663-673

Hannon, B. \& Joyce, J. (1981). Energy and Technical Progress. Energy, 6(2), 187-195.

Henderson, F. (1933). The Economic Consequences of Power Production. London: George Allen \& Unwin Ltd. 
Manrique-Díaz y Lemus-Polanía: Procedimiento de optimización no lineal...

Humphrey, W. S., and Stanislav, J. (1979). Economic growth and energy consumption in the UK, 1700-1975. Energy Journal, 7(1), 29-42.

Hwang, D. \& Gum, B. (1991). The causal relationship between energy and GNP: the case of Taiwan, The Journal of Energy and Development, 16(2), 219-226.

Jensen, W. G. (1970). Energy and the Economy of Nations, Atlanta: G.T. Foulis and Co, Henley-on-Thames.

Jorgenson, D. W. (1978). The Role of Energy in the U. S. Economy, National Tax Journal, 31(3), 209-220.

Jorgenson, D. W. (1981). Energy Prices and Productivity Growth, The Scandinavian Journal of Economics, 83(2), 165-179.

Jorgenson, D. (1984). The role of energy in productivity growth, The Energy Journal, 5(3), pp. 11-26

Kalmanovitz, S. (2010). Nueva historia económica de Colombia, Bogotá D.C.: Taurus - UJTL.

Kraft, J. \& Kraft, A. (1978). On the relationship between energy and GNP, Journal of Energy and Development, 3(2), 401-403.

Kümmel, R. (1980). Growth Dynamics of the Energy Dependent Economy, Cambridge: Oelgeschlager, Gun \& Hain.

Kümmel, R. (1982). The impact of energy on industrial growth. Energy, 7(2), 189-203.

Kümmel, R. (1989). Energy as factor of production and entropy as a pollution indicator in macroeconomic modeling, Ecological Economics, 1(2), 161-180.

Kümmel, R., Strassl, W., Gossner, A. \& Eichhorn, W. (1985). Technical progress and energy dependent production functions, Zeitschrift für Nationalökonomie Journal of Economics, 45(3), 285-311. 
Kümmel, R., Lindenberger, D. \& Eichhorn, W. (2000). The productive power of energy and economic evolution, Indian Journal of Applied Economics, $8(2), 231-262$.

Kümmel, R., Henn, J. \& Lindenberger, D. (2002). Capital, labor, energy, and creativity: modeling innovation diffusion, Structural Change and Economic Dynamics, 13(4), 415-433

Kümmel, R., Ayres, R. \& Lindeberger, D. (2010). Thermodynamics laws, economic methods and the productive power of energy, Journal of NonEquilibrium Thermodynamics, 35(2), 145-179.

Kümmel, R. (2011). The Second Law of Economics: Energy, Entropy, and The Origins of Wealth, New York: Springer.

Kümmel, R. y Lindenberger, D. (2014), How energy conversion drives economic growth far from the equilibrium of neoclassical economics, New Journal of Physics, 16(12), 1-21.

Kümmel, R., Lindenberger, D. \& Weiser, F. (2015). The economic power of energy and the need to integrate it with energy policy, Energy Policy, 86, 833-843.

Lee, C.-C. (2006). The casuality relationship between energy comsuption and GDP in G-11 countries revisited, Energy Policy, 34(9), 1086-1093.

Lee, C.-C. \& Chang, C.-P. (2007). Energy consumption and GDP revisited: a panel analysis of developed and developing countries, Energy Economics, 29(6), 1206-1223.

Lindenberger, D. \& Kümmel, R. (2011). Energy and the state of nations, Energy, 36(10), 6010-6018.

Lindenberger, D., Weiser, F., Winkler, T. y Kümmel, R. (2017). Economic Growth in the USA and Germany 1960-2013: The Underestimated Role of Energy, Biophysical Economics and Resource Quality, 2(3), 1-23.

Lotka, A. J. (1922). Contribution to the energetics of evolution. Proceedings of the National Academy of Sciences; 8, 147-151. 
Manrique-Díaz y Lemus-Polanía: Procedimiento de optimización no lineal...

Nachane, D., Nadkarni, R. \& Karnik, A. (1988). Co-integration and causality testing of the energy-GDP relationship: a cross-country study, Applied Economics, 20(11), 1511-1531.

Nahman, A. \& Antrobus, G. (2005). The environmental Kuznets curve: a literature review. South African Journal of Economics, 73(1), 105-120.

Narayan, P.-K. \& Popp, S. (2012). The energy consumption-real GDP nexus revisited: empirical evidence from 93 countries, Economic Modelling, 29(2), 303-308.

Nordhaus, W. D. (2008). A Question of Balance: Weighing the Options on Global Warming Policies, New Haven, Connecticut: Yale University Press.

Ocampo, J. A. \& Montenegro, S. (1982). La crisis mundial de los años treinta en Colombia, Desarrollo y Sociedad, 7(1), 37-95.

Oh, W. y Lee, K. (2004). Casual relationship between energy conpsumption and GDP revisited: the case of Korea 1970-1999, Energy Economics, 26(1), 51-59.

Ostwald, W. (1907). The Modern Theory of Energetics. The Monist, 17(4), 481-515.

Ozturk, I., Aslan, A. \& Kalyoncu, H. (2010). Energy consumption and economic growth relationship: Evidence from panel data for low and middle income countries, Energy Policy, 38(8), 4422-4428.

Pokrovski, V. (2003). Energy in the theory of production, Energy, 28(8), 769-788.

Pokrovski, V. (2007). Productive energy in the US economy, Energy, 32(5), 816-822.

Ricci, F. (2007). Channels of transmission of environmental policy to economic growth: a survey of the theory. Ecological Economics, 60(4), 688-699.

Rifkin, J. (2011). La Tercera Revolución Industrial, Barcelona: Paidós. 
Schurr, S. H. \& Netschert, B. C. (1960). Energy in the American Economy, 1850-1975 (Series). Baltimore, MD, Johns Hopkins University Press.

Schurr, S., Burwell, C., Devine, W. \& Sonenblum, S. (1990). Electricity in the American Economy: Agent of technological progress, Santa Barbara: Greenwood Praeger

Sharma, S.-S. (2010). The relationship between energy and economic growth: Empirical evidence from 66 countries, Applied Energy, 87(11), 3565-3574.

Shengfeng, X., Sheng, X.-M., Tianxing, Z. \& Xuelli, Z. (2012). The relationship between Electricity Consumption and Economic Growth in China, Physics Procedia, 24(Part A), 56-62.

Shiu, A. \& Lam, P.-L. (2004). Electricity consumption and economic growth in China, Energy Policy, 32(1), 47-54.

Shuyun, Y. \& Donghu, Y. (2011). The causality between energy consumption and economic growth in China: using panel method in a Multivariate Framework, Energy Procedia, 5, 808-812.

Smil, V. (2005). Energy and the Crossroads: Global Perspectives and Uncertainties, Cambridge: MIT Press.

Soddy, F. (1912). Matter and Energy. London: Oxford University Press.

Solow, R. M. (1956). A contribution to the theory of economic growth, The Quarterly Journal of Economics, 70(1), 65-94.

Solow, R. M. (1957). Technical Change and the Aggregate Production Function, The Review of Economics and Statistics, 39(3), 312-320.

Solow, R. M. (1994). Perspectives on Growth Theory, The Journal of Economic Perspectives, 8(1), 45-54.

Soytas, U. \& Sari, R. (2003). Energy consumption and GDP: Causality relationship in G-7 countries and emerging markets, Energy Economics, 25(1), 33-37. 
Manrique-Díaz y Lemus-Polanía: Procedimiento de optimización no lineal...

Squalli, J. (2007). Electricity consumption and economic growth: bounds and causality analyses of OPEC members, Energy Economics, 29(6), 1192-1205.

Stern, D. I. (1993). Energy and economic growth in the USA: a mutivariate approach, Energy Economics, 15(2), 137-150.

Stern, D. I. (2004). Economic Growth and Energy. Encyclopedia of Energy, 2, $35-51$.

Tyron, F. G. (1927). An Index of Consumption of Fuels and Water Power, Journal of The American Statistical Association, 22(159), 271-282.

Yalta, T. \& Cakar, H. (2012). Energy consumption and economic growth in China: A reconciliation, Energy Policy, 41, 666-675.

White, L. A. (1943). Energy and the evolution of culture. American Anthropologist, 45(3, Part 1), 335-356.

Wolde-Rufael, Y. (2009). Energy consumption and economic growth: The experience of African countries revisited, Energy Economics, 31(2), 217-224.

Yildirim, E. \& Aslan, A. (2012). Energy consumption and economic growth nexus for 17 highly developed OECD countries: Further evidence based on bootstrap-corrected causality tests, Energy Policy, 51, 985-993.

Yoo, S.-H. (2006). The casual relationship between electricity consumption and economic growth in the ASEAN countries, Energy Policy, 34(18), 3573-3582.

Yu, E. \& Choi, J.-Y. (1985). The casual relationship between energy and GNP: An international comparison, Journal of Energy and Development, 10(2), 249-272.

Yuan, J.-H., Kang, J.-G., Zhao, C.-H. \& Hu, Z.-G. (2008). Energy consumption and economic growth: Evidence from China at both aggregated and disaggregated levels, Energy Economics, 30(6), 3077-3094. 
Zhang, C. y Xu, J. (2012). Retesting the causality between energy consumption and GDP in China: Evidence from sectoral and regional analyses using dynamic panel data, Energy Economics, 34(6), 1782-1789. 